\title{
Investigation of the Flow Characteristics of Droplets in Non-uniform Electric field
}

\author{
Zhonghua Gao \\ School of Electric Power Engineering, North China Electric Power University, Baoding \\ 071000, China. \\ 1194892568@qq.com
}

Key Words: Non-uniform electric field, droplets, deformation, Numerical simulation.

\begin{abstract}
The motion behavior of droplets under non-uniform electric field is an important branch the study of electro-hydrodynamic. In this paper, the theoretical analysis of the force of droplets in non-uniform electric field are carried out, and the study found that the deformation, surface charge density, velocity and other flow characteristics of droplets will change in the effect of non-uniform electric field.

In order to study those flow characteristics and the influence of electric field intensity on the deformation and displacement degree of droplets, we will establish the simulation model of droplets and the non-uniform electric field.The results show that the polarization charge distribution on the surface of droplets is not uniform, and the charge density at the center of the electric field is increased gradually; the increase of the electric field intensity in a certain range can increase the droplet shape variable and the moving velocity of the droplet to the concentrated area of the electric field.
\end{abstract}

\section{Introduction}

The electric field is the fundamental driving force for the liquid drop deformation. Under the non-uniform electric field, the droplets is deformed along the direction of the electric field line and the displacement occurs at the same time to the field intensity concentration. The relevant scholars have carried out the research on the movement behavior of the droplets under the uniform electric field .For example,the dynamics of deformation and rupture of a droplet in uniform electric field is studied by Kazimierz ${ }^{[1]}$ etc. However, previous studies did not involve the movement behavior of liquid droplet deformation and displacement under the action of non-uniform electric field.

Therefore, in this paper, based on the level set method of two-phase flow field in Multiphysics COMSOL 4.4, simulation model is established to study the distribution of the potential, electric field, droplets and the velocity vectors around the fluid and the deformation and displacement behaviors of droplets.

\section{Model Assumptions}

To simplify the problem, we make the following assumptions :

- The molecular shape of droplet is spherica.

- Fluid is an incompressible ideal fluid.

- Assuming that there are other fluids around the droplet, the droplet is subjected to the viscous effect of the surrounding fluid.

\section{Theoretical analysis in non-uniform electric field}

\section{Force of droplets in a non-uniform electric field}

The droplet molecule can be regarded as an electric dipole, the electric dipole moment: $p_{e}=q l$ (1)

Where 1 represents the distance between the point charge. As is shown in Figure 1 below, the electric field force is less than 0 in the non-uniform electric field: 


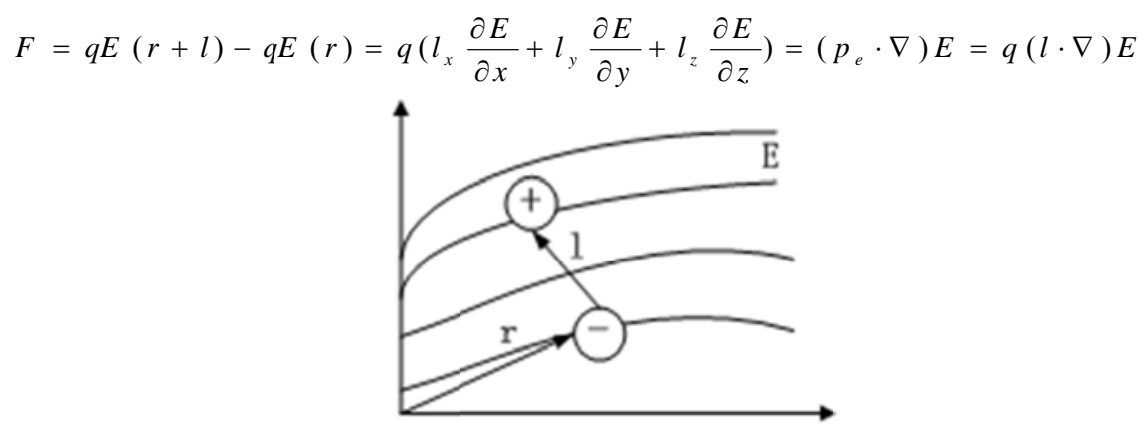

Fig.1 Force of an electric droplet in a non-uniform electric field

Force of per unit volume of liquid in a non-uniform field:

$$
f=n F=\left(n p_{e} \cdot \nabla\right) E=(P \cdot \nabla) E=\left[\varepsilon_{0}\left(\varepsilon_{r}-1\right) E \cdot \nabla\right] E=\frac{1}{2} \varepsilon_{0}\left(\varepsilon_{r}-1\right) \nabla E^{2}
$$

Where $\mathrm{n}$ represents liquid molecular density, $\varepsilon_{0}$ represents permittivity of vacuum, $\varepsilon_{r}$ represents relative permittivity.

By the formula above, the force size of the unit volume is proportional to the gradient of the square of the electric field intensity.

\subsection{Analysis of the motion behavior of droplets in a non-uniform electric field}

- Under the non-uniform electric field, the shape of the droplet is transformed from spherical to ellipsoidal and the droplets is deformed along the direction of electric field line and displaced to the field intensity concentration.

- In the process of deformation, the liquid droplets are subjected to the viscosity of the surrounding fluid.

\section{Droplet Simulation model}

\section{Droplet Simulation model}

Assuming that the droplet morphology is spherical,the simulation model:liquid drop suspended in oil is as follows:

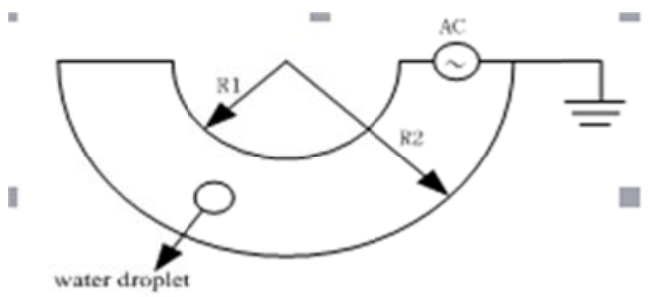

Fig.2 Simulation model of water droplets suspended in oil

\section{Initialization of the parameters}

Table 1 Simulation of the input parameters of droplet

\begin{tabular}{ccc}
\hline Material parameter & Numerical value & Unit symbol \\
\hline Droplet diameter ( $\mathrm{AC})$ & 4.0 & $\mathrm{~mm}$ \\
electrode voltage & 400 & $\mathrm{~V} / \mathrm{mm}$ \\
Initial velocity of water droplet & 5.0 & $\mathrm{~mm} / \mathrm{s}$ \\
Droplet density $\rho_{A}$ & 1000 & $\mathrm{~kg} / \mathrm{m}^{3}$ \\
Oil density $\rho_{1}$ & 880 & $\mathrm{~kg} / \mathrm{m}^{3}$ \\
Water droplet conductivity k & 5.5 & $\mu \mathrm{S} \cdot \mathrm{m}^{-1}$ \\
\hline
\end{tabular}




\section{Non-uniform electric field Simulation model}

In order to study the deformation and movement law of the droplet under the non-uniform electric field, the simulation analysis was carried out by the multi field coupling software Multiphysics COMSOL 4.4.

Non-uniform electric field model-Coaxial cylinder electrode model

The electrode is connected to the external electrode, the inner electrode is grounded. $(\mathrm{R} 1=5 \mathrm{~mm}$, $\mathrm{R} 2=10 \mathrm{~mm})$
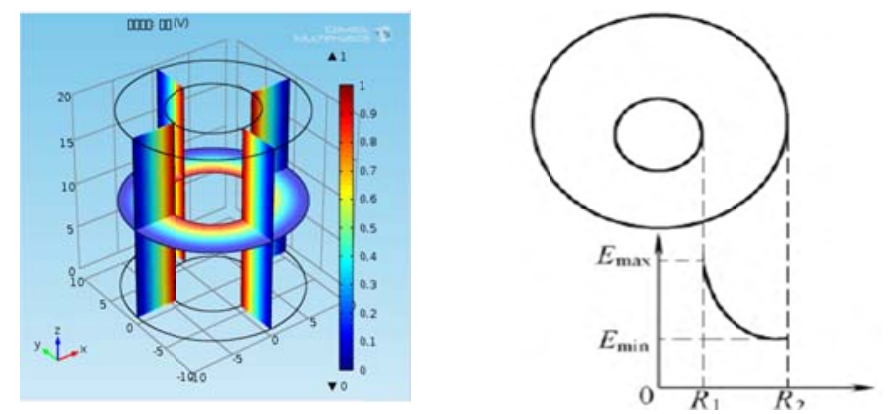

Fig.3 Simulation results of coaxial cylindrical electrodes

As is shown in fig.3, the distribution of electric potential is consistent with electric field intensity, and along the radius direction away from the center to the far, the electric potential is lower.

Interior and exterior electric potential distribution of droplets

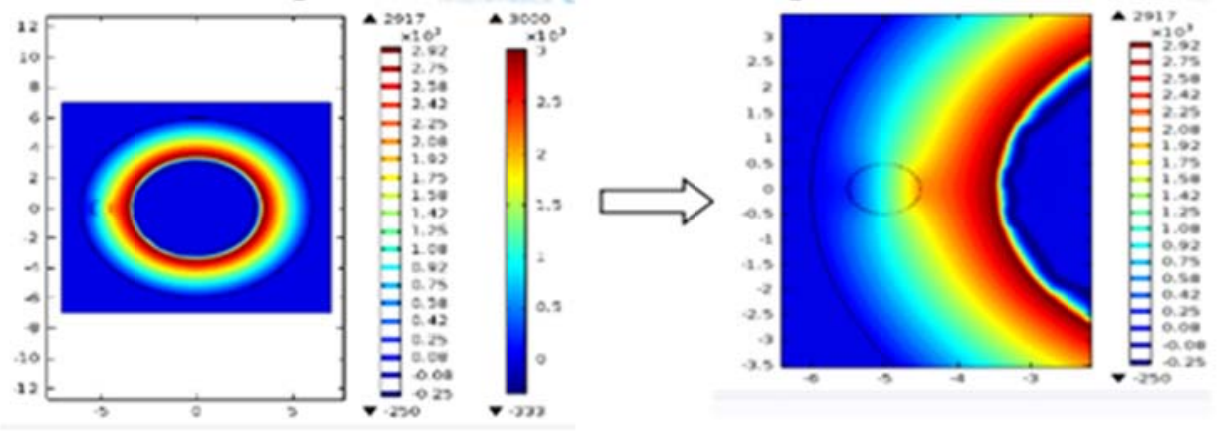

Fig.4 Interior and exterior electric potential distribution of droplets

From the above diagram, the internal and external electric potential distribution have a certain law : Along the radius direction away from the center to the far, the electric potential is lower.

\section{Droplet deformation and displacement}

Deformation $^{[2]}$ and displacement of the droplet in $\mathrm{t}=0 \mathrm{~s}$ to $\mathrm{t}=0.1 \mathrm{~s}$ periodic are simulated in the following figure.

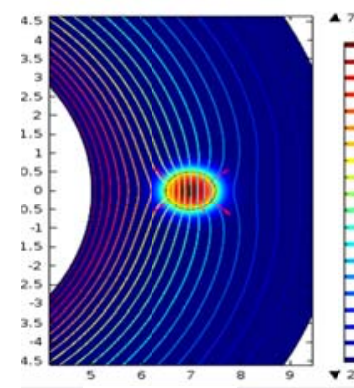

$\mathrm{t}=0 \mathrm{~s}$

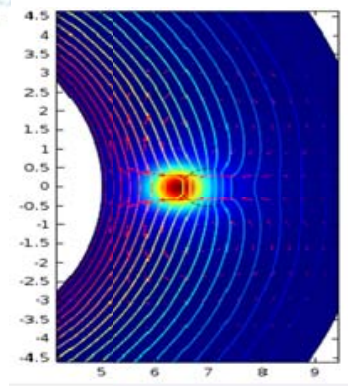

$\mathrm{t}=0.05 \mathrm{~s}$
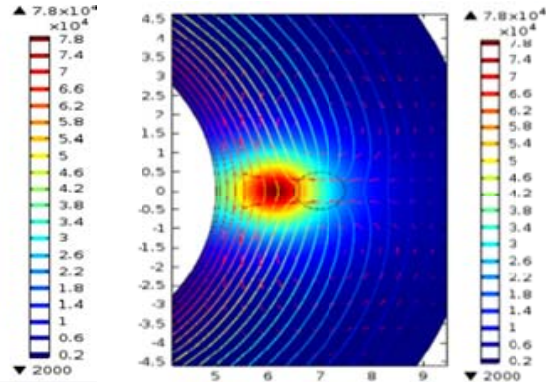

$\mathrm{t}=0.1 \mathrm{~s}$

Fig.5 Single droplet deformation

As is shown above,the droplet is gradually extended from spherica to ellipsoidal. Which shows that the droplet is deformed along the direction of the electric field line and the displacement occurs at the same time to the field intensity concentration. 


\section{Deformation under different electric field intensity}

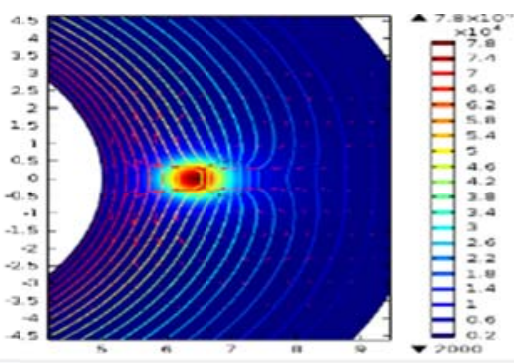

$\mathrm{E}=400 \mathrm{~V} / \mathrm{mm}$

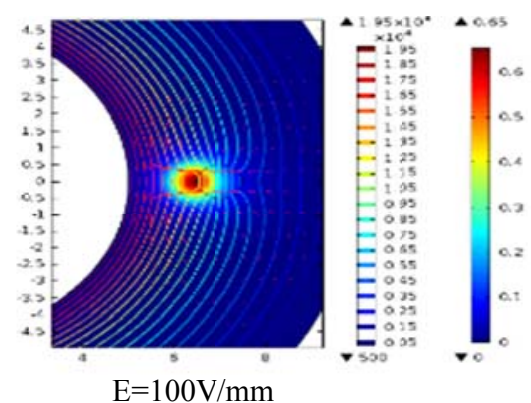

$\mathrm{E}=100 \mathrm{~V} / \mathrm{mm}$

Fig.6 Deformation under different electric field intensity

As is shown in Fig.6,with the increase of electric field intensity,the maximum deformation degree of droplets increase also. When the field intensity increases to a certain degree, the deformation degree is too large and even rupture. ${ }^{[3]}$

\section{Summary}

(1)Under non-uniform electric field, the polarization charge density is gradually increased from the middle to both ends of the droplet, and at the end of the concentration of electric field intensity to reach the maximum.

(2)The droplet is deformed along the direction of the electric field line and the displacement occurs at the same time to the field intensity concentration.

(3)With the increase of electric field intensity, the deformation degree of droplets increase also. And the droplet will be ruptured under high electric field intensity.

\section{References}

[1]Kazimierz Adamiak, Jerzy M. Floryan, "Dynamics of water droplet distortion and breakup in a uniform electric field.” IEEE T. IND. APPL, vol. 47, pp. 2374-2382, 2011.

[2] Zhang Jun, He Hongzhou, Huang Guanxing. Simulation of droplet deformation in uniform electric field with dissipative particle dynamics approach[J].CIESC Journal,2014,65(10): 3872-3877.

[3]Chen Qingguo, Liang Wen, Song Chunhui. Effect of electric field strength on crude oil emulsion's demulsification and dehydration[J]. High Voltage Engineering, 2014, 40 (1):173-180 